\title{
Characterizations Based on Cumulative Entropy of the Last-order Statistics
}

\author{
Osman Kamari ${ }^{1}$ \\ ${ }^{1}$ College of Administration and Economics, University of Human Development, Sulaymaniyah, Iraq \\ Correspondence: Osman Kamari, College of Administration and Economics, University of Human Development, \\ Sulaymaniyah, Iraq. E-mail: osman.kamari@uhd.edu.iq
}

Received: November 22, 2016

Accepted: April 19, 2017 Online Published: April 23, 2017

doi:10.5539/ijsp.v6n3p183

URL: https://doi.org/10.5539/ijsp.v6n3p183

\begin{abstract}
Cumulative Entropy (CE) as a measure of uncertainty alternative to Shannon entropy is proposed by Di Crescenzo and Longobardi (2009). In this paper, some properties of the cumulative entropy are derived and under conditions are showed the cumulative entropy of the last order statistics can determine the distribution function uniquely. Weibull family is characterized by ratio of the cumulative entropy of the last order statistics to its expectation. Also, some inequalities are presented for the cumulative entropy of reversed residual lifetime of a parallel system.
\end{abstract}

Keywords: cumulative entropy, shannon entropy, last order statistics, weibull family, reversed residual lifetime

\section{Introduction}

Shannon (1948) introduced entropy, known Shannon entropy, as a measure of uncertainty for a random variable. Suppose $X$ be a non-negative continuous random variable having probability density function $f$ and cumulative distribution function $F$. Hence, Shannon entropy $h(X)$ of a continuous non-negative random variable $X$ with probability density function $f(x)$ is defined as

$$
h(X)=-\int_{0}^{+\infty} f(x) \log f(x) d x
$$

Di Cresceno and Longbardi (2002) introduced the entropy of the reversed residual lifetime ${ }_{t} X=[t-X \mid X<t]$ as a dynamic measure of uncertainty as follows

$$
H(X ;[t])=-\int_{0}^{t} \frac{f(x)}{F(t)} \log \frac{f(x)}{F(t)} d x
$$

It's clear that $\mathrm{H}(\mathrm{X} ; \infty)=h(X)$.

Rao et al. (2004) defined Cumulative Residual Entropy(CRE) as an alternative measure of uncertainty to Shannon entropy in that the probability density function is replaced by survival(reliability) function and obtained some properties and applications of that(2005). Rao showed CRE overcomes some problems with Shannon entropy. Some properties of CRE are: CRE is more general rather than the Shannon entropy, it has more mathematical properties rather than Shannon entropy, and it is easily computed from sample data. CRE has applications in reliability engineering and image processing, for more details see Rao $(2004,2005)$. CRE for a non-negative univariate random variable is as follows:

$$
\operatorname{CRE}(X)=-\int_{0}^{\infty} \bar{F}(x) \log \bar{F}(x) d x .
$$

That $\bar{F}(x)=P(X>x)=1-F(x)$ is the survival function of $X$.

Crescenzo and Longobardi (2009) introduced a new information measure that turns out to be useful to measure information on the reversed residual lifetime of a system. The reversed residual lifetime is a concept in reliability that is convenient to describe the time elapsing between the failure of a system and the time when it is down. This measure is defined as follows:

$$
C E(X)=-\int_{0}^{\infty} F(x) \log F(x) d x
$$

where $F(x)$ is the cumulative distribution function of the non-negative random variable $X$. Also, they proposed dynamic form of cumulative entropy that called dynamic cumulative entropy and obtained some of its properties. 
Cumulative entropy for reversed residual lifetime with distribution function $F_{t}(x)=P[t-X \mid X<t]$ is

$$
C E(X ; t)=-\int_{0}^{t} F_{t}(x) \log F_{t}(x) d x=-\int_{0}^{t} \frac{F(x)}{F(t)} \log \frac{F(x)}{F(t)} d x
$$

It's clear $C E(X ; \infty)=C E(X)$.

By using probability integral transformation $F(x)=U$ that $U$ have uniform distribution function on $(0,1)$, then we obtain

$$
C E(X)=-\int_{0}^{1} \frac{u \log u}{f\left(F^{-1}(u)\right)} d u
$$

That $F^{-1}$ is inverse function of $F$.

Suppose $X_{1}, X_{2}, \ldots, X_{n}$ be a random sample, the order statistics of the sample is defined by the arrangement $X_{1}, X_{2}, \ldots, X_{n}$ from the minimum to the maximum by $X_{1: n}, X_{2: n}, \ldots, X_{n: n}$. Order statistics are used in entropy estimation, quality control, reliability, insurance, etc. For more details see Arnold et al. (1992), David and Nagaraja (2003).

Baratpour (2010) have derived characterizations result based on Cumulative residual entropy of first order statistics. Also Thapliyal et al. (2013) studied cumulative entropy and dynamic cumulative entropy using order statistics. They showed a characterization property that dynamic cumulative entropy of the $i$ th order statistics determines the distribution function. The purpose of this paper is characterization result the parent distribution based on Cumulative Entropy of the last order statistics.

The paper is organized as follows: In section 2, characterizing the parent distributions based on the CE of the last order statistics is presented. Also, Weibull distribution is characterized based on the ratio of the CE of the last order statistics to the expectation of it. Last section includes some Characterizations based on CE of reversed residual lifetime of parallel systems.

\section{Characterizations Based on the Last-order Statistics}

Last order statistics is an important special case of order statistics. Suppose $X_{n: n}$ be the last order statistics in a random sample of size $\mathrm{n}$, cumulative distribution function of $X_{n: n}$ is $F_{X_{n: n}}(x)=(F(x))^{n}$, Thus CE of $X_{n: n}$ is defined as follow:

$$
C E\left(X_{n: n}\right)=-n \int_{0}^{\infty}(F(x))^{n} \log F(x) d x
$$

By changing variable $F(x)=U$, we have

$$
C E\left(X_{n: n}\right)=-n \int_{0}^{1} \frac{u^{n} \log (u)}{f\left(F^{-1}(u)\right)} d x
$$

In the following theorem, we show that only in Weibull family the ratio of the CE of the last order statistics to the expectation of it is constant. First we need a lemma taken from Baratpour (2010).

Lemma 2.1: For any increasing sequence of positive integers $\left\{n_{j}, j \geq 1\right\}$, the sequence of polynomials $\left\{x^{n_{j}}\right\}$ is complete on $\mathrm{L}(0,1)$, if and only if $\sum_{j=1}^{\infty} \frac{1}{n_{j}}$ be infinitely.

Theorem 2.1: Let $X_{1}, X_{2}, \ldots, X_{n}$ are positive, independent and identically(iid) distributed observations from an absolutely continuous cumulative distribution function $F(x)$ and probability density function $f(x)$. Then $F(x)$ belongs to Weibull family if and only if $\frac{C E\left(X_{n: n}\right)}{E\left(X_{n: n}\right)}=c(>0)$ for all $n=n_{j}, j \geq 1$ such that $\sum_{j=1}^{\infty} \frac{1}{n_{j}}$ be infinitely.

Proof: The 'if' part of the theorem is trivial. Hence we prove 'only if' part, using integral transformation $F(x)=U$ in 


$$
E\left(X_{n: n}\right)=\int_{0}^{\infty} n x f(x)[F(x)]^{n-1} d x=n \int_{0}^{1}[F(u)]^{-1} u^{n-1} d u
$$

according to relations (2) and (3) we obtain

$$
\frac{C E\left(X_{n: n}\right)}{E\left(X_{n: n}\right)}=-\frac{\int_{0}^{1} \frac{u^{n} \log (u)}{f\left(F^{-1}(u)\right)} d u}{\int_{0}^{1}[F(u)]^{-1} u^{n-1} d u}
$$

Relation (4) coincides c, so we have

$$
\begin{aligned}
& \int_{0}^{1} \frac{u^{n} \log (u)}{f\left(F^{-1}(u)\right)} d u+c \int_{0}^{1}[F(u)]^{-1} u^{n-1} d u=0 \\
& \int_{0}^{1} u^{n-1}\left[\frac{u \log u}{f\left(F^{-1}(u)\right)}+c F^{-1}(u)\right] d u=0
\end{aligned}
$$

If relation (5) holds for $n=n_{j}, j \geq 1$ such that $\sum_{j=1}^{\infty} \frac{1}{n_{j}}=\infty$, then from lemma 2.1 we have

$$
\frac{(1-v) \log (1-v)}{f\left(F^{-1}(1-v)\right)}+c F^{-1}(1-v)=0
$$

Because $\frac{d F^{-1}(1-v)}{d v}=\frac{1}{f\left(F^{-1}(1-v)\right)}$ then it follows:

$$
(1-v) \log (1-v) \frac{d F^{-1}(1-v)}{d v}+c F^{-1}(1-v)=0 \quad v \in(0,1)
$$

By solving this differential equation, we have

$$
\frac{\frac{d F^{-1}(1-v)}{d v}}{F^{-1}(1-v)}=-\frac{c}{(1-v) \log (1-v)}
$$

$\mathrm{F}^{-1}(1-v)=\mathrm{c}_{1}[-\log (1-\mathrm{v})]^{\mathrm{c}}, \mathrm{v} \in(0,1)$ thus $\mathrm{F}(\mathrm{x})=1-\exp \left\{-\left(\frac{\mathrm{x}}{\mathrm{c}_{1}}\right)^{\frac{1}{\mathrm{c}}}\right\}, \mathrm{x}>0$ we conclude that $\mathrm{F}(\mathrm{x})$ belongs to the Weibull family.

Theorem 2.2: Suppose $X, Y$ be two positive random variables with probability density functions $f(x)$ and $g(x)$ and absolutely continuous distribution functions $F(x)$ and $G(x)$, respectively. Then $F$ and $G$ belong to the same family of distributions, but for a location shift, if and only if

$$
C E\left(X_{n: n}\right)=C E\left(Y_{n: n}\right)
$$

for $n=n_{j}, j \geq 1$ such that $\sum_{j=1}^{\infty} \frac{1}{n_{j}}$ be infinitely.

Proof: The 'if' part of the theorem is trivial. Hence we prove 'only if' part, according to relation (2) if $C E\left(X_{n: n}\right)=$ $C E\left(Y_{n: n}\right)$, then

$$
\int_{0}^{1} u^{n} \log u\left[\frac{1}{f\left(F^{-1}(u)\right)}-\frac{1}{g\left(G^{-1}(u)\right)}\right] d u=0
$$

if for all $n=n_{j}, j \geq 1$ such that $\sum_{j=1}^{\infty} \frac{1}{n_{j}}=\infty$, then from Lemma 2.1 we can conclude that

$$
f\left(F^{-1}(t)\right)=g\left(G^{-1}(t)\right), \quad 0<t<1 .
$$


Because $\frac{d F^{-1}(t)}{d t}=\frac{1}{f\left(F^{-1}(t)\right)}$, we have $\frac{d F^{-1}(t)}{d t}=\frac{d G^{-1}(t)}{d t}, 0<t<1$, then $F^{-1}(t)=G^{-1}(t)+d, 0<t<1$. this means for a change of location parameter, $F$ and $G$ belong to the same family.

\section{Characterizations Based on Cumulative Entropy of Reversed Residual Lifetime of Parallel Systems}

Suppose $X_{1}, X_{2}, \ldots, X_{n}$ be continuous and iid random variables with common cumulative distribution function $F$ denote the lifetimes of $n$ components of a parallel system. Also $X_{1: n}, X_{2: n}, \ldots, X_{n: n}$ be the ordered lifetimes of the components. Then $X_{n: n}$ represents the lifetime of that parallel system with cumulative distribution function $F_{X_{n: n}}(x)=(F(x))^{n}, x>0$. The distribution function of $\left[t-X_{n: n} \mid X_{n: n}<t\right]$ is $F_{X_{n: n}}(x)=\left(\frac{F(t-x)}{F(t)}\right)^{n}$ that $\left[t-X_{n: n}\right]$ is called reversed residual lifetime of system. Now cumulative entropy for reversed residual lifetime of parallel system with distribution function $F_{X_{n: n}}(x)$ is as follows:

$$
\begin{aligned}
& C E\left(X_{n: n, t}\right)=-\int_{0}^{\infty} X_{n: n, t} \log X_{n: n, t} d x=-\int_{0}^{t}\left(\frac{F(x)}{F(t)}\right)^{n} \log \left(\frac{F(x)}{F(t)}\right)^{n} d x \\
= & -\frac{1}{(F(t))^{n}} \int_{0}^{t}(F(x))^{n} \log (F(x))^{n} d x+n \log F(t) \int_{0}^{t}\left(\frac{F(x)}{F(t)}\right)^{n} d x \\
= & -\frac{1}{(F(t))^{n}} \int_{0}^{t}(F(x))^{n} \log (F(x))^{n} d x+n \log F(t) K_{X_{n: n}}(t)
\end{aligned}
$$

That $K_{X_{n: n}}(t)=E\left(t-X_{n: n} \mid X_{n: n}<t\right)$ is the mean reversed residual lifetime of the system.

Now according to relation $(7)$ and that $\log F(t) \leq 0$, we obtain some lower bounds for $C E\left(X_{n: n}\right)$ as follows:

$$
C E\left(X_{n: n, t}\right) \leq-\frac{1}{(F(t))^{n}} \int_{0}^{t}(F(x))^{n} \log (F(x))^{n} d x \leq-\frac{1}{(F(t))^{n}} \int_{0}^{\infty}(F(x))^{n} \log (F(x))^{n} d x=\frac{1}{(F(t))^{n}} C E\left(X_{n: n}\right)
$$

hence, for all $\mathrm{t}$ we have

$$
C E\left(X_{n: n}\right) \geq(F(t))^{n} C E\left(X_{n: n, t}\right)
$$

According to non-negativity of $C E$ and relation (7) we obtain

$$
\begin{aligned}
K_{X_{n: n}}(t) \leq- & \frac{1}{n \log |F(t)|(F(t))^{n}} \int_{0}^{t}(F(x))^{n} \log (F(x))^{n} d x \leq-\frac{1}{n \log |F(t)|(F(t))^{n}} \int_{0}^{\infty}(F(x))^{n} \log (F(x))^{n} d x \\
& \leq-\frac{1}{n \log |F(t)|(F(t))^{n}} C E\left(X_{n: n}\right)
\end{aligned}
$$

Finally

$$
C E\left(X_{n: n}\right) \geq n \log |F(t)|(F(t))^{n} K_{X_{n: n}}(t)
$$

Theorem 3.1: Suppose $X$ and $Y$ be two positive random variable with density functions $f(x)$ and $g(x)$ and absolutely continuous cumulative distribution functions $F(x)$ and $G(x)$, respectively. Then $F$ and $G$ belong to the same family of distributions, but for a change in location and scale parameters, if and only if for $t>0$

$$
C E\left(X_{n: n, t}\right)=C E\left(Y_{n: n, t}\right)
$$

for $n=n_{j}, j \geq 1$ such that $\sum_{j=1}^{\infty} \frac{1}{n_{j}}$ be infinitely. 
Proof: The 'if' part of the theorem is obvious. So to prove 'only if' part, if for all $n=n_{j}, j \geq 1, \sum_{j=1}^{\infty} \frac{1}{n_{j}}=\infty$, $C E\left(X_{n: n, t}\right)=C E\left(Y_{n: n, t}\right)$, then according to theorem (2.2) $[X \mid X<t]$ and $[Y \mid Y<t]$ have the same distribution but for a location shift, means $f_{t}(x)=g_{t}(x+c)$ that $f_{t}, g_{t}$ are respectively density functions of $[X \mid X<t]$ and $[Y \mid Y<t]$.

Hence $f(x)=\frac{F(t)}{G(t)} g(x+c)$ that is $F$ and $G$ belong to the same family of distributions but for a change in location and scale parameters.

\section{Refrences}

Arnold, B. C., Balakrishnan, N., \& Nagaraja, H. N. (1992). A First Course in Order Statistics. New York: John Wiley \& Sons.

Bratpour, S. (2010). Characterizations Based on Cumulative Residual Entropy of First-Order Statistics. Communications in Statistics - Theory and Methods, 39(20) 3645 -3651. https://doi.org/10.1080/03610920903324841

David, H. A., \& Nagaraja,. N. H (2003). Order Statistics. 3rd ed. New York, John Wiley \& Sons. https://doi.org/10.1002/0471722162

Di Crescenzo, A., \& Longobardi, M. (2002). Entropy- based measure of uncertainty in past lifetime distributions, J. Appl. Probab,. 39, 434-440. https://doi.org/10.1017/S002190020002266X

Di Crescenzo, A., \& Longobardi, M. (2009). On cumulative entropies. Journal of Statistical Planning and Inference, 139, 4072-4087. https://doi.org/10.1016/j.jspi.2009.05.038

Rao, M. (2005). More on a new concept of entropy and information. J. Theoret. Probab. 18, 967-981. https://doi.org/10.1007/s10959-005-7541-3

Rao M., Chen Y., Vemuri B. and Wang F. (2004). Cumulative residual entropy: a new measure of information. IEEE Transactions on Information Theory, 50, 1220-1228. https://doi.org/10.1109/TIT.2004.828057

Shannon, C. E. (1948). A mathematical theory of communication. Bell System Technical J. 27. 379-423. https://doi.org/10.1002/j.1538-7305.1948.tb01338.x

Thapliyal, R, Kumar, V., \& Tanej, H. C., (2013). On dynamic cumulative entropy of order statistics. Journal of Statistics Applications \& Probability, 2(1), 41-46. https://doi.org/10.12785/jsap/020106

\section{Copyrights}

Copyright for this article is retained by the author(s), with first publication rights granted to the journal.

This is an open-access article distributed under the terms and conditions of the Creative Commons Attribution license (http://creativecommons.org/licenses/by/4.0/). 